\section{Algunas reflexiones sobre la formación en la universidad}

ALGUNOS DE LOS TEMAS que constantemente, de una forma u otra, están saliendo en los periódicos estos últimos meses hacen referencia a la formación universitaria. Y lo hacen por una parte desde la perspectiva de si las futuras titulaciones de grado, con el enfoque del Espacio Europeo de Educación Superior, se adaptarán realmente a las necesidades del mercado laboral. Y por otra está la discusión sobre los aspectos referidos al propio gobierno de las universidades. Especialmente si deberían tener más intervención por parte de la sociedad, en especial por parte de los ámbitos de la empresa, de la administración y de la política, para conferirle un "enfoque de utilitarismo".

Referente al primer punto y dentro de nuestro ámbito enfermero, cabe decir que las Escuelas de Enfermería siempre han tenido en cuenta las demandas de las instituciones sanitarias, y asimismo suelen contar con profesorado de las propias instituciones de salud para la realización de algunos seminarios y la tutorización de las prácticas clínicas.

Por otra parte, la mayoría del profesorado está realizando innovaciones en las diferentes materias que componen el plan de estudios de grado, a pesar de que los recursos no se han incrementado y el número de alumnos ha aumentado de acuerdo con los criterios establecidos por la universidad; por ejemplo, en lo que se refiere al mínimo número de alumnos para la participación en los seminarios y en las clases prácticas. En general tampoco se ha incrementado el profesorado, a pesar de que se han puesto en marcha los programas de másters universitarios y de doctorado. Esperemos que con todos los esfuerzos que se están realizando y al contar con un curso académico más, los futuros graduados dispongan de una formación próxima a las necesidades de los usuarios del sistema sanitario, pero también de una formación global y humanista que les confiera una actitud crítica y positiva con el sistema de salud y social, y que a la par promueva avances confiriendo valor a la profesión enfermera.

Por lo que respecta al segundo punto, cabe tener en cuenta que la universidad no es una empresa de formación. Una universidad reducida a empresa no sirve a los ideales de verdad y desarrollo de la cultura y a la formación de pensadores. Si fuera así, se limitaría a producir profesionales medianamente útiles a la sociedad. La universidad es una institución académica que, naturalmente, en los diferentes programas de formación de las distintas titulaciones ha de tener en cuenta las necesidades y las demandas del mercado laboral, pero al tratarse de una institución de rango académico superior, esta formación no es suficiente.

Se espera que a la par que el estudiante adquiere competencias sobre la propia profesión, se forme también en los valores estrechamente ligados a la formación universitaria, como son la búsqueda de la verdad; la honestidad como valor que conduce al conocimiento y a las limitaciones del saber, la acción y las propias carencias; la solidaridad que equivale al compromiso con la sociedad; el respeto a la vida y a los demás como factor de cohesión social y también el respeto a la naturaleza como conciencia ecológica; la responsabilidad como asunción de compromisos; la justicia como medio de procurar la mejor distribución e igualdad de oportunidades; la integridad como ejemplo de rectitud; la libertad como autodeterminación y elección de alternativas; los principios éticos, entre muchos otros. Por tanto, la universidad como portadora de valores es creadora de una cultura en la que, además del conocimiento técnico, deben imperar el humanismo, la investigación, la innovación y la transferencia, entre otros aspectos.

En consecuencia, gobernar la universidad bajo unos criterios que no sean los puramente académicos conlleva riesgos importantes y puede desdibujar esta formación de excelencia que la sociedad espera. De todas formas, seguro que eso no quiere decir que no sea necesario, al igual que han hecho otros sectores de la sociedad, profesionalizar la gestión de forma que el profesorado que quiera optar a cargos académicos tenga conocimientos expertos en los ámbitos de planificación estratégica, de organización y gestión, de conducción de grupos, etc.

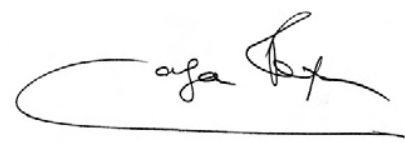

Margarita Peya Gascóns Directora Nursing edición española
EDITORIAL ADVISORY BOARD

Los miembros del Advisory Board ejercen como revisores y proporcionan los contenidos editoriales de la revista.

Elizabeth A. Ayello, RN, APRN,BC, PhD, FAAN, FAPWCA, President, Ayello, Harris \& Associates, Inc.; Faculty, Excelsior College School of Nursing, Albany, N.Y.; Senior Adviser, The John A. Hartford Institute for Geriatric Nursing and Program Director, Education Essentials, New York, N.Y.

Mary M. Bailey, RN, CEN, CMSRN, BSN, Clinical Educator, Albert Einstein Medical Center, Philadelphia, $\mathrm{Pa}$

Penny Simpson Brooke, APRN, MS, JD, Professor and Director of Outreach, University of Utah College of Nursing, Salt Lake City, Utah

Helen Calabretto, RN, RM, Dip T (N Ed), B Ed (Nsg St), M Ed St, PhD, FRCNA, Senior Lecturer, School of Nursing and Midwifery, University of South Australia, Adelaide, South Australia

Michael R. Cohen, RPh, MS, ScD, President, Institute for Safe Medication Practices, and Member of the Sentinel Event Advisory Group for The Joint Commission, Huntingdon Valley, $\mathrm{Pa}$.

Yvonne D'Arcy, CRNP, CNS, MS, Pain Management and Palliative Care Nurse Practitioner, Suburban Hospital, Bethesda, Md.

Michael W. Day, RN, CCRN, MSN, Outreach Trauma Nurse-Coordinator, Sacred Heart Medical Center and Children's Hospital,Spokane, Wash.

Jeanette Ives Erickson, RN, MS, Senior Vice-President for Patient Care and Chief Nurse, Massachusetts General Hospital, Boston, Mass.

Martha M. Funnell, RN, CDE, MS, Codirector of the Behavioral, Clinical and Health Systems Intervention Research Core, Michigan Diabetes Research and Training Center; Research Investigator in the Department of Medical Education; and Adjunct Lecturer, School of Nursing, University of Michigan, Ann Arbor, Mich

Peg Gray-Vickrey, RN,C, DNS, Professor of Nursing, and Associate VicePresident of Curriculum and Instruction, Florida Gulf Coast University, Fort Myers, Fla.

Lynn C. Hadaway, RN,C, CRNI, MEd, President, Lynn Hadaway Associates, Inc., Milner, Ga.

Jeanne Held-Warmkessel, RN, AOCN, APRN,BC, MSN, Clinical Nurse Specialist, Fox Chase Cancer Center, Philadelphia, $\mathrm{Pa}$

Susan Simmons Holcomb, ARNP,BC, PhD, Family NP, Olathe, Kan. Patrick E. Kenny, RN, ACRN, CNA,BC,C, EdD, Director of Nursing Education and Research, Penn Presbyterian Medical Center (University of Pennsylvania Health System), Philadelphia

Joan E. King, RN,C, ACNP, ANP, PhD Program Director for Acute Care Adult Nurse Practitioners, Vanderbilt University School of Nursing, Nashville, Tenn.

Linda Laskowski-Jones, RN, APRN,BC, CCRN, CEN, MS, VicePresident, Emergency, Trauma, and Aeromedical Services, Christiana Care Health System, Wilmington, Del.

Paul J. Mathews, RRT, PhD, FAARC, FCCM, FCCP, Associate Professor of Respiratory Care, University of Kansas Medical Center, Kansas City, Kan.

Frank Edward Myers III, CIC, MA Director of Clinical Epidemiology and Safety Systems, Scripps Mercy Hospital, San Diego, Calif.

Bill Pruitt, RRT, AE-C, CPFT, MBA Senior Instructor and Director of Clinical Education, Cardiorespiratory Sciences, School of Allied Health, University of South Alabama, Mobile, Ala.

Richard L. Pullen, Jr., RN, EdD, Professor of Nursing and Assistant Director of ADN Program, Amarillo College, Amarillo, Tex.

Kathleen Reeves, RN, CMSRN, CNS, MSN Clinical Assistant Professor, University of Texas Health Science Center at San Antonio; President, Academy of Medical-Surgical Nurses, San Antonio, Tex.

Sylvia Rodgers, RN, BScN, MEd, Professional Practice Leader, Center for Nursing, Scarborough Hospital, Toronto, Ontario

Suzanne C. Smeltzer, RN, EdD, FAAN, Professor and Director, Center for Nursing Research, Villanova University College of Nursing, Villanova, Pa.

LeAnn Thieman, LPN, Motivational Speaker and Writer, author of Chicken Soup for the Nurse's Soul, Second Dose and 12 other books, Fort Collins, Colo.

Deborah Tuggle, RN, CCNS, MN, Critical Care Clinical Nurse Specialist, Jewish Hospital, Louisville, Ky. 